\title{
Die Kultur von Halicystis parvula (Codiales, Chlorophyta) auf kalkhaltigem Substrat
}

\author{
P. Kornmann \\ Biologische Anstalt Helgoland (Meeresstation); \\ D-2192 Helgoland, Bundesrepublik Deutschland
}

\begin{abstract}
The culture of Halicystis parvula (Codiales, Chlorophyta) on calcareous substratum. The vesicular thallus of Halicystis is generally embedded in crusts of coralline algae by a rhizoidal part. Only in former unsuccessful attempts to elucidate the development of Halicystis has this fact been regarded as being important. Starting from Derbesia zoospores, the gametophyte Halicystis was attained in free culture. These vesicles, however, are unlike those of the natural plants in morphological aspect: they develop as a local increase of a rhizoidal filament. To grow into vesicles of natural shape in culture, the zoospores of Derbesia need a calcareous substratum, e. g. fragments of oyster-shell.
\end{abstract}

\section{EINLEITUNG}

Der blasige Thallus von Halicystis ist im allgemeinen mit einem Rhizoid in Corallinaceen-Krusten verankert. Printz (1926) fand ein einzelnes Exemplar auf einem SerpulaGehäuse, eine für die hier angewandte Methode interessante Angabe. In den früheren Versuchen, die Entwicklung von Halicystis aufzuklären, wurde die Beziehung zum Substrat als wesentlich angesehen. Kuckuck (1907) versuchte, die "Makrozoosporen" von Halicystis ovalis auf Lithothamnion-Krusten zur Keimung zu bringen. Hollenberg (1935) ließ ihre Zygoten auf natürlichem Substrat keimen und erhielt eine kriechende, fädige Generation; seine Interpretation der direkten Entstehung von Halicystis aus deren in das Substrat eingedrungenen Rhizoiden trifft aber nicht zu. Von Derbesia marina-Zoosporen ausgehend, wurde Halicystis ovalis in freier Kultur erhalten (Kornmann, 1938); damit war der heteromorphe Generationswechsel aufgeklärt und zugleich auch erwiesen, daß eine obligate Bindung des Gametophyten an das Substrat nicht besteht. Jedoch entsprechen die in freier Kultur entstandenen Halicystis-Blasen morphologisch nicht den natürlich gewachsenen. Sie entwickeln sich als lokale - nicht immer apikale - Anschwellung eines mehrere Millimeter langen fädig-rhizoidalen, mitunter auch verzweigten Keimlings; die Blasen tragen daher meist einen unnatürlichen, kurzen Fortsatz.

In allen folgenden Untersuchungen, die von Halicystis parvula oder Derbesia tenuissima ausgingen (Feldmann, 1950; Köhler, 1958; Rietema, 1973), blieb die Bedeutung des Substrats für die Entstehung der Halicystis-Phase unberücksichtigt. In der vorliegenden Studie wurden den Derbesia-Zoosporen Muschelschalenfragmente als Substrat angeboten. Die Erwartung bestätigte sich: die so entstandenen HalicystisBlasen entsprechen ihrer natürlichen Form. 


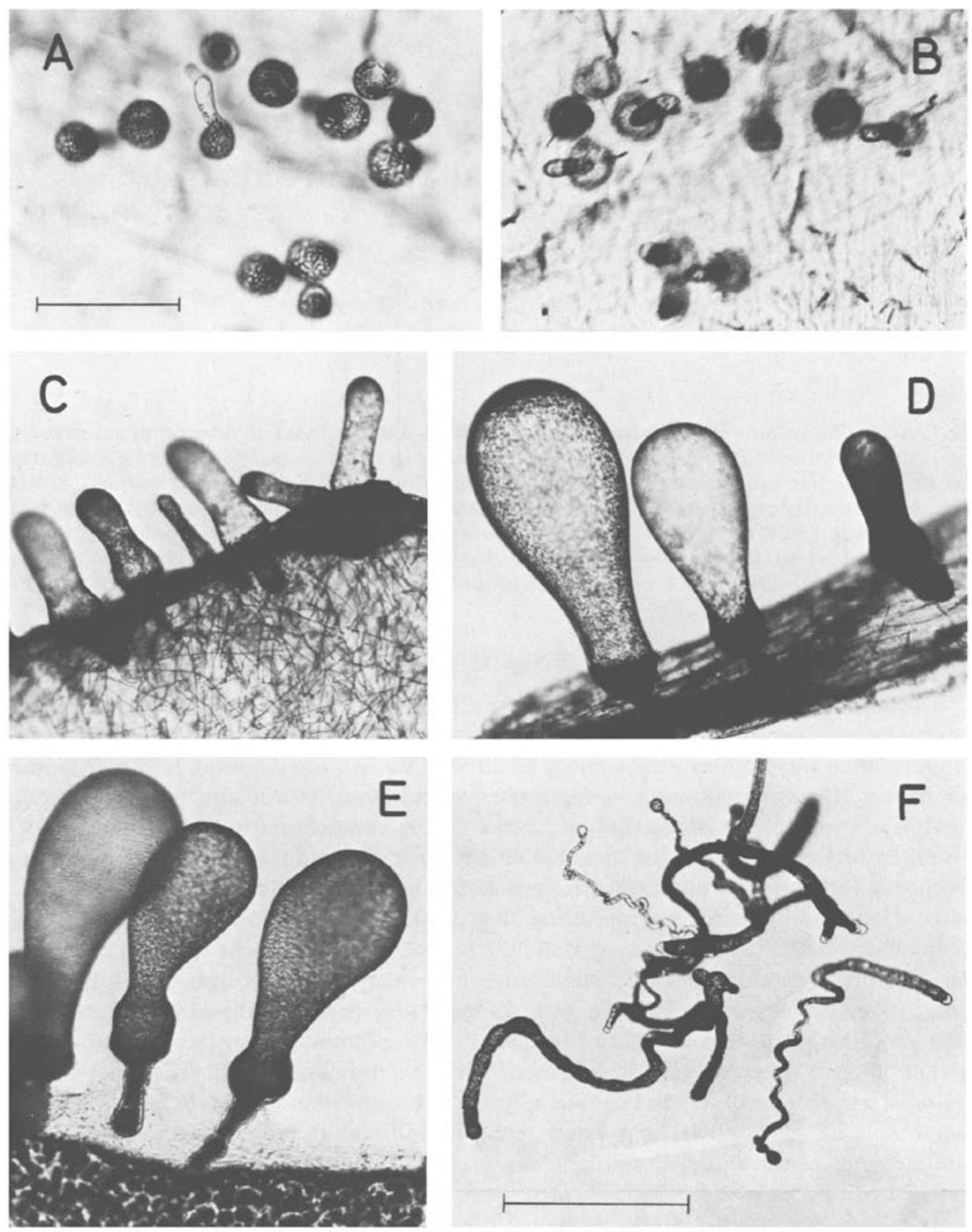

Abb. 1. Derbesia tenuissima, Entwicklung der Halicystis-Phase. A, B 5 Tage alte ZoosporenKeimlinge auf einem Austernschalenfragment, dieselbe Gruppe von oben und von unten gesehen. C Etwa 20 Tage alte Halicystis an der Kante eines Schalenfragments. D, E Etwa 4 Wochen alte Halicystis, lebend in einem Schalenfragment und nach Auflösung des Substrats. F 4 Wochen alte Halicystis-Pflanzen in freier Kultur. Maßstrecken: $A, B=100 \mu \mathrm{m} ; C-F=500 \mu \mathrm{m}$ 


\section{MATERIAL UND METHODE}

Für die Versuche stand mir der Stamm LB 1260 von Derbesia tenuissima aus der Kulturensammlung der Universität Texas durch Herrn Prof. Kies, Hamburg, zur Verfügung. Sporophyt und Gametophyt gedeihen gut in Erdschreiberlösung und in Nährlösung nach Provasoli mit einem Zusatz von Erdabkochung bei $15^{\circ} \mathrm{C}$ und 14 stündiger Beleuchtung bei etwa 2000-2500 Lux. Als Substrat wurden wegen ihrer guten Durchsichtigkeit meist Austernschalenfragmente verwendet; es werden aber auch Schalen von Cardium, Wohnröhren von Pomatoceros, Balanus-Platten und tote LithothamnionKrusten als Keimbett angenommen.

\section{ERGEBNISSE UND DISKUSSION}

Das Ergebnis der unter diesen Kulturbedingungen durchgeführten Versuche sei vorweggenommen; die auf den Muschelschalen zur Ruhe gekommenen Zoosporen entwickelten sich rasch zu normal gestalteten Halicystis-Blasen, während die gleichzeitig auf dem Schalenboden oder an der Oberfläche der Flüssigkeit wachsenden Keimlinge rhizoidal blieben $(\mathrm{Ab} b, 1 \mathrm{E}, \mathrm{F})$. Eine Gruppe von 5 Tage alten Keimlingen auf einem dünnen Schalenfragment ist in der Aufsicht (A) und von unten betrachtet dargestellt (B). Die sehr unterschiedlich großen Zoosporen setzen sich auf ihrem zilientragenden Pol fest. Aus der Mitte der recht großen Ansatzfläche senkt sich ein dünner Rhizoidschlauch in das Substrat ein. Dann erst wölbt sich die junge Halicystis-Blase über der ursprünglichen Zoospore vor, bleibt aber zunächst noch deutlich von ihr abgesetzt. Etwa 20 Tage alte Halicystis-Blasen sind schlauchförmig, meistens sogar apikal etwas verjüngt (C). Danach erfolgt das rasche Wachstum zur typischen ovalen Form von Halicystis parvula (D, E). 7 Wochen alte Gametophyten waren bis zu $5 \mathrm{~mm}$ hoch und wurden fertil.

Halicystis ist in der Natur eine obligat kalkbohrende Alge. In Kultur entwickelt sie sich nur zu ihrer typischen Form, wenn die Derbesia-Zoosporen auf einem entsprechenden Substrat keimen. In freier Kultur entstandene rhizoidale Keimlinge können nicht mehr in Kalk eindringen.

Das Substrat ist für die normale Entwicklung von Halicystis von ebenso entscheidender Bedeutung wie der Einfluß der Tageslänge. Bei den gewählten Versuchsbedingungen $-15^{\circ} \mathrm{C}$ und 14 stündiger Beleuchtung - entstehen in freier Kultur keine blasigen Pflanzen (Abb. $1 \mathrm{~F}$ ). Rietema (1973) untersuchte die Entstehung der Halicystis parvulaBlasen bei $20{ }^{\circ} \mathrm{C}$ in Abhängigkeit von der Beleuchtungsdauer. Er erhielt blasige Halicystis bei 16 Stunden Lichtgenuß, während bei 8stündiger Beleuchtung nur rhizoidal-fädige Pflänzchen entstanden.

Daß die Entstehung der Halicystis-Blasen wesentlich von der Tageslänge abhängt, zeigen schon meine Versuche mit Derbesia marina (Kornmann, 1938). Sie wurden nicht unter kontrollierten Bedingungen, sondern in einem Raum bei Tageslicht in der Zeit von August 1937 bis Mai 1938 durchgeführt. Erst mit zunehmender Tageslänge begannen die rhizoidalen Keimlinge Mitte März zu kugeliger Halicystis heranzuwachsen.

Danksagung. Meinem Mitarbeiter, Herrn Paul-Heinz Sahling, danke ich für das stetige Interesse, mit dem er die Entstehung dieser Arbeit verfolgte und für die Anfertigung der photographischen Belege. 


\section{ZITIERTE LITERATUR}

Feldmann, J., 1950. Sur l'existence dune alternance de générations entre l'Halicystis parvula Schmitz et le Derbesia tenuissima (De Not.) Crn. - C. r. hebd. Séanc. Acad. Sci., Paris (D) 230. $322-323$

Hollenberg, G. J., 1935. A study of Halicystis ovalis. I. Morphology and reproduction. - Am. J. Bot. $22,782-812$.

Köhler, K., 1958. Uber den Generationswechsel von Halicystis-Derbesia im Golf von Neapel. Pubbl. Staz, Zool. Napoli 30, 342-346.

Kornmann, P., 1938. Zur Entwicklungsgeschichte von Derbesia und Falicystis. - Planta 28, $464-470$.

Kuckuck, P., 1907. Uber den Bau und die Fortpflanzung von Halicystis Areschoug und Valonia Ginnani. - Bot. Ztg 65, 139-185.

Printz, H., 1926. Die Algenvegetation des Trondhjemsjfordes. - Skr. norske VidenskAkad. 1926, (5), $1-274$.

Rietema, H., 1973. The influence of day length on the morphology of the Halicystis parvula phase of Derbesia tenuissima (De Not.) Crn. (Chlorophyceae, Caulerpales). - Phycologia 12, 11-16. 